\title{
Designing Web Sites and Interfaces to Optimize Successful User Interactions: Symposium Overview
}

\author{
Robert W. Proctor ${ }^{1}$ and Kim-Phuong L. Vu ${ }^{2}$ \\ ${ }^{1}$ Department of Psychological Sciences, Purdue Univerity, West Lafayette, IN, USA \\ ${ }^{2}$ Department of Psychology, California State Univerity Long Beach, Long Beach, CA, USA \\ proctor@psych.purdue.edu, kvu8@csulb.edu
}

\begin{abstract}
Since the Web became widely available in the mid 1990s, it has come to be used by a range of people for many purposes. Effective user interactions are required for a Web site or product to accomplish its intended goals. Given the user-intensive nature of the Web and the many usability issues associated with performing tasks on the Web and with mobile devices, it is important for designers and researchers to understand issues that relate to how to optimize interfaces for the Web design and other systems involving humancomputer interaction. This symposium is devoted to issues involved in the design of Web sites and interfaces to promote successful user interactions.
\end{abstract}

Keywords: Information Display, Input Devices, Mobile Devices, Organization of Information, Web Design.

\section{Introduction}

Since the World Wide Web became widely available in the mid 1990s, it has come to be used by a variety of people for many different purposes, including e-commerce, social networking, data display, information sharing and collaboration, and mobile transactions. Effective user interactions are required for a Web site or product to accomplish its intended goals. Given the user-intensive nature of the Web and the numerous usability issues associated with performing tasks on the Web and with mobile devices, designers and researchers need to understand issues relating to Web design and Web usability. There is often a tendency to pit academicians against practitioners, basic research against applied research, and theoretical knowledge against experiential knowledge. Yet, from our experience, we have found that an approach that emphasizes multiple perspectives and multiple methods is most beneficial for acquiring knowledge and advancing technology [1]. Communication among individuals with various backgrounds, interests, and training is essential for facilitating the development and transfer of knowledge between researchers and practitioners in the domain of human-computer interaction (HCI), among other applied domains. Because the most complete understanding of usability problems arises from combining the insights of practitioners and in industry and government with the knowledge of academicians gained from controlled research, we consistently strive to encourage interaction among experts from the different communities. 
To that end, in 2005, we edited the Handbook of Human Factors in Web Design [2], in which we articulated the general view described above specifically for Web design, stating:

It is our opinion that the handbook should reflect the varied backgrounds and interests of individuals involved in all aspects of human factors and Web design. Consequently, we have made a concerted effort to obtain contributions from a diverse group of researchers and practitioners. The contributors are an international ensemble of individuals from academia, industry, and research institutes. Furthermore, the contributors have expertise in a variety of disciplines. We think that awareness of the wide range of views and concerns across the field is essential for usability specialists and Web designers, as well as for researchers investigating theoretical and applied problems concerning Web use and design. (p.xv)

Consistent with this view, the book covered a full range of topics relevant to human factors in Web design, from historical developments and human factors principles in general to specific human factors applications in Web design. These content areas included content preparation for Web sites, search engines and interface agents, issues of universal accessibility, usability engineering, Web applications in academic and industrial settings, information security, and emerging technologies such as wireless communication and e-health.

That same year, we also organized a symposium for HCII 2005, "Human Factors Considerations in Web and Application Design", which highlighted many of the then current issues concerning usability in Web design. Papers presented in the symposium included: "Web-based Presentation of Information: The Top Ten Mistakes and Why They Are Mistakes" [3], "User Search Strategy in Web Searching" [4], "Cross Cultural Web Design" [5], "Understanding Online Consumers: A More Complete Picture" [6], "Web-based Programs and Applications for Business" [7], and "User Interface Design Guidelines" [8].

All of the topics covered in the 2005 handbook and symposium are still relevant today, but technological and societal developments have fueled many changes in Web use since that time. Social networking was in its infancy, mobile computing was being developed, and 4G wireless was not available. Rapid developments in these areas, as well as others, have greatly changed the computing landscape, and all of the developments involve new human factors issues associated with use of the technology. To capture these developments, we have edited a second edition of the handbook, which was recently published [9]. Because most of the topics that were relevant in 2005 continue to be relevant today, many chapters of the second edition provide updated information on those topics. New chapters are devoted to topics that have emerged as important since the first edition. They include: Organization of Information for Concept Sharing and Web Collaboration, Web-Based Organization Models, Web Portals, Human Factors of Online Games, Accessibility Guidelines and the ISO Standards, Use of Avatars, and Mobile Interface Design for M-Commerce.

Much as the 2005 symposium was intended as an adjunct to the first edition of the handbook, this 2011 symposium is intended to be a companion to the recently published second edition, highlighting a subset of usability topics of interest to 
designers and researchers interested in the Web and HCI. Fitting with our emphasis on communication among academicians and practitioners, the papers represent contributions from persons working in industry and academia.

\section{Overview of Symposium}

This symposium is devoted to issues involved in organization and display of information for HCI in general and Web design in particular. The first two papers address user interactions on e-commerce sites. Najjar presents "Advances in Ecommerce User Interface Design," in which he emphasizes that interface design is not a static field but a dynamic one in which possible interface features to incorporate into designs are continually changing. He describes new user interface features of which ecommerce designers may want to take advantage when designing Web sites. Examples are provided for many features, including social media connections, automated product recommendations, contextual product visualization, flash sales, and mobile commerce. When performing e-commerce transactions, personal information is transmitted through the Web. The topic of privacy is addressed by Nguyen and $\mathrm{Vu}$ in their paper, "Does Privacy Information Influence Users' Online Purchasing Behavior?" In their study, users indicated whether they would make a purchase of an inexpensive or expensive item at different Web sites. Privacy information was made salient by a search engine called PrivacyFinder, but this information did not appear to influence users' purchasing behaviors.

Pappas and Whitman, in their paper "Riding the Technology Wave: Effective Dashboard Data Visualization," discuss how to optimize data dashboard displays, identifying the types of data that are best represented in particular formats and techniques for displaying multiple visualizations. They emphasize that the choice of what to display and how to display it depends on the needs of the particular user. In "A Conceptual Model of Using Axiomatic Evaluation Method for Usability of the Consumer Electronics," Guo, Proctor, and Salvendy describe concepts from Axiomatic Design theory that are based in information theory, and they discuss how it can be applied as a usability evaluation method for mobile consumer electronics. An experiment is described in which participants identified more usability problems associated with a cell phone when employing axiomatic evaluation than when using a more traditional usability method.

Mobile devices are also of concern to Xu and Bradburn, whose paper, "Usability Issues in Introducing Capacitive Interaction into Mobile Navigation," focuses on user interactions with mobile devices. They present an experiment that evaluates the use of capacitive touch sensors that are able to distinguish light and forceful touches, as a possible option for interface design. Xu and Bradburn discuss issues associated with implementation of capacitive touch devices and propose initial guidelines for their use. In the final paper, "Movement Time for Different Input Devices," Bacon and Vu describe an experiment showing movement times for three input devices commonly used in HCI tasks. Movement time was shortest when the input modality was a button press on a response panel, intermediate when it was a computer mouse, and slowest when it was a touch screen. The authors discuss implications for the design of display-control configurations using these input devices. 
This symposium contains both basic and applied knowledge derived from experiments and design experience. Each paper provides a unique contribution to understanding issues for optimizing interfaces and Web sites for human use.

\section{References}

1. Proctor, R.W., Vu, K.-P.L.: Complementary Contributions of Basic and Applied Research in Human Factors and Ergonomics. Theor. Iss. in Erg. Sci. (in press)

2. Proctor, R.W., Vu, K.-P.L. (eds.): Handbook of Human Factors in Web Design. Lawrence Erlbaum Associates, Mahwah (2005)

3. Tullis, T.: Web-based Presentation of Information: The Top Ten Mistakes and Why They Are Mistakes. In: HCI International 2005, Human-Computer Interfaces: Concepts, New Ideas, Better Usability, and Applications, vol. 3. Lawrence Erlbaum Associates, Mahwah, (2005)

4. Fang, X.: User Search Strategy in Web Searching. In: HCI International 2005, HumanComputer Interfaces: Concepts, New Ideas, Better Usability, and Applications., vol. 3. Lawrence Erlbaum Associates, Mahwah (2005)

5. Rau, P.-P.P., Choong, Y.-Y., Plocher, T.: Cross Cultural Web Design. In: HCI International 2005, Human-Computer Interfaces: Concepts, New Ideas, Better Usability, and Applications, vol. 3. Lawrence Erlbaum Associates, Mahwah (2005)

6. Volk, F., Kraft, F.: Understanding Online Consumers: A More Complete Picture. In: HCL 2005, Human-Computer Interfaces: Concepts, New Ideas, Better Usability, and Applications, vol. 3. Lawrence Erlbaum Associates, Mahwah (2005)

7. Vaughan, M., Dumas, J.: Web-based Programs and Applications for Business. In: HCL 2005, Human-Computer Interfaces: Concepts, New Ideas, Better Usability, and Applications, vol. 3. Lawrence Erlbaum Associates, Mahwah (2005)

8. Najjar, L.: Accessible Java-Application User Interface Design Guidelines. In: International, H.C.I. (ed.) HCI International 2005, Human-Computer Interfaces: Concepts, New Ideas, Better Usability, and Applications, vol. 3. Lawrence Erlbaum Associates, Mahwah (2005)

9. $\mathrm{Vu}$, K.-P.L., Proctor, R.W. (eds.): Handbook of Human Factors in Web Design, 2nd edn. CRC Press, Boca Raton (2011) 